\title{
Using MAS to Detect Retinal Blood Vessels
}

\author{
Carla Pereira ${ }^{1}$, Jason Mahdjoub ${ }^{2}$, Zahia Guessoum², Luís Gonçalves ${ }^{3}$, \\ Manuel Ferreira ${ }^{1}$ \\ ${ }^{1}$ Industrial Electronics Department, University of Minho, Campus de Azurém, 4800-058 \\ Guimarães, Portugal \\ ${ }^{2}$ CReSTIC - MODECO, University of Reims, rue des Crayères, 51100, Reims, France \\ ${ }^{3}$ Ophthalmology Service, Centro Hospitalar do Alto Ave, Guimarães, Portugal
}

\begin{abstract}
The segmentation of retinal vasculature by color fundus images analysis is crucial for several medical diagnostic systems, such as the diabetic retinopathy early diagnosis. Several interesting approaches have been done in this field but the obtained results need to be improved. We propose therefore a new approach based on an organization of agents. This multi-agent approach is preceded by a preprocessing phase in which the fundamental filter is an improved version of the Kirsch derivative. This first phase allows the construction of an environment where the agents are situated and interact. Then, edges detection emerged from agents' interaction. With this study, competitive results as compared with those present in the literature were achieved and it seems that a very efficient system for the diabetic retinopathy diagnosis could be built using MAS mechanisms.
\end{abstract}

Keywords. Diabetic retinopathy; Fundus images; Image processing; Kirsch filter; Multi-agent system

\section{Introduction}

The segmentation of the retinal vasculature is of major importance for clinical purposes. In fact, by analyzing the vascular structures it is possible to have the early diagnosis of several chronic pathologies, such as diabetic retinopathy (DR). In this study, the blood vessels segmentation was per- 
formed concerning this pathology that has been revealed as the leading cause of vision impairment in developed countries. In order to prevent loss of vision it is important to detect the pathology as early as possible.

At present, the most common way to analyze vascular modifications is the digital color fundus photograph as it is a non invasive technique. Numerous research efforts have been done in segmenting the blood vessels by image processing techniques applied in the fundus images [1]-[6]. The main difficulties in accurately segment the vessels are due to the presence of pathologies, noise, the low contrast between vasculature and background, the variability of vessels width, brightness and shape. To solve this problem of variability, it is important to locally adapt interpretations on the image instead of applying only one algorithm on the entire image. A multi-agent system (MAS) is thus proposed as a solution since agents allow the cohabitation of several algorithms. In fact, agents can analyze problems they are locally confronted with, and select the most suitable algorithm to their local context [7].

There are some works described in the literature that associate MAS with image processing in medical images [7]-[12]. Different MAS proprieties and mechanisms have been used in these approaches, such as interaction, self-organization, adaptation, communication, cooperation and negotiation [12]. Richard et al. [9] proposed a hierarchical architecture of situated and cooperative agents to segment the cerebral tissue in MRI. Duchesnay et al. [10] present an approach where the agents are organized as an irregular pyramid and cooperate to aggregate regions. Bovenkamp et al. [11] proposed a MAS for the segmentation of the IntraVascular UItraSound images. The main characteristic of this approach is the elaboration of a high-level knowledge-based control over the low-level image processing algorithms.

Therefore, the association of MAS and image processing has been revealed as an expanded area of research. As far as we know, multi-agent approaches have never been applied to the retinal images. In this paper, an approach based on the previous work of Mahdjoub et al. [7] is applied to the digital color fundus images for the blood vessels segmentation. Despite of the wide literature in the segmentation of blood vessels trough fundus images analysis [1]-[6], none of them performs as needed due to 
the use of centralized mechanisms at the macro level. Since the retinal images are very complex, it is necessary to develop algorithms that can be locally adapted to the image proprieties. This new approach uses some image processing algorithms as concrete perception and action tools for defining autonomous agents that interact among themselves and with the environment (the image). Then the segmentation of the blood vessel emerges as a global behavior.

This paper is organized as follows: Section 2 describes the proposed approach compounded of two main phases: the preprocessing and the MAS model. The results are demonstrated and discussed in Section 3. Finally, conclusion is presented in Section 4 .

\section{Methodology}

The proposed approach aims to use a MAS model to improve the detection of retinal blood vessels edges resulting from a preprocessing phase. This preprocessing phase consists of a group of conventional image processing algorithms and provides the information (environment) for the MAS model reconstructs and re-organizes them.

\subsection{Preprocessing}

For this step of the approach, the methods used in the preprocessing phase of work developed by Niemeijer et al. [13] were first employed. The resultant image had no intensity variation in the background across the image and the bright structures were eliminated. A Gaussian filter (width 3 pixels; $\sigma=2$ ) was then applied to attenuate the high frequency noise.

In order to remove the noise of the fundus image while preserving the edges it was then applied the Kuwahara filter [14]. Finally to the image resulted from the last step a modified version of the Kirsch filter was employed. The Kirsch filtering is a method for enhancing the edges in an image using a basic convolution filter rotated eight times. All eight filters are applied to the image with the maximum being retained for the final im- 
age. The improved Kirsch filter [7] enables to detect edges with a two pixels thickness of which the external edge is represented by a positive or negative value, whereas the internal edge has an opposite value (Fig. 1 a)). This facilitates the detection process of the MAS model since the gradient of the blood vessels reveals a specific pattern (Fig. 1 b)) as they can be represented by series of two parallel linear segments. Thus, the agents search the edges of the blood vessels by looking for this specific pattern of the gradient.

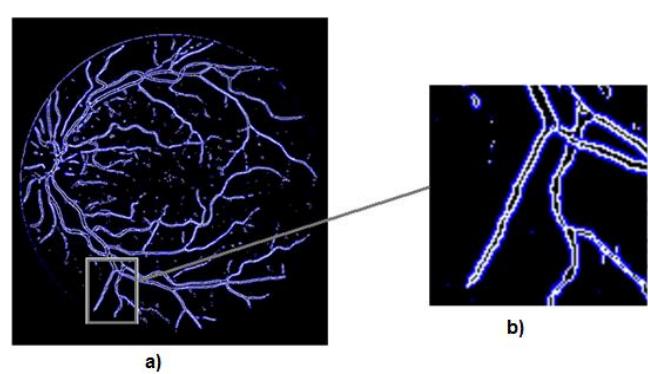

Fig. 1. a) Resultant image of the modified Kirsch filter where the blue and white pixels represent negative and positive gradient values, respectively. b) Expanded version of a section of image a), where is possible to see a characteristic pattern in the vessel gradient values.

\subsection{A Multi-Agent Approach for the Edge Detection}

Our MAS is composed by a set of agents and their environment. The environment contains the green plane image in which each pixel contains the grey level of intensity and a boolean value defining if the pixel has already been explored by an agent. Moreover, when located in the environment the agents perceive the Kirsch gradient which defines a right visible edge. The agents are of several kinds with different behaviors according to their current state and perception: search agent (SA), following agent (FA), node agent (NA), and end agent (EA). The algorithm initializes with a search agent launched on one of the white points from Fig. 1 a), randomly chosen. At the end of the process the agents have to reconstruct the vessels edge by representing it with a succession of segments. 


\section{Agents}

The SA initially launched by the system has to find edges and evolves in the environment by following the points with positive gradient. When it finds an edge, it determines the possible directions to follow the contour, creates a NA and moves to another position. The NA creates FAs in the directions given by the SA and establishes segments with them. The FAs follow the edge detected until its segment doesn't correspond with the explored contour anymore. Then they create NAs, give them information about the direction to follow and die. These new NAs create new FAs, and so on. Considering that several agents can follow the same contour, they end up meeting and merging. When there is no direction to follow the NA creates an EA.

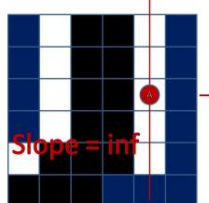

a)

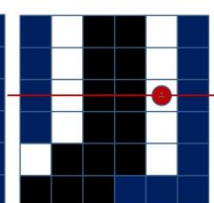

b)

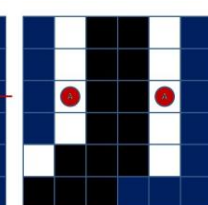

c)

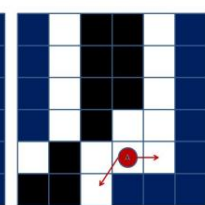

d)

Fig. 2. The SA (red circle) behavior. a) It calculates the slope of the line to determine the perpendicular line (b). Then it verifies the gradient values profile and as the pixel belongs to a vessel pattern it launches another SA (c); d) Possible directions (red arrows) that the agent has to follow according to the search agents' restrictions

Search behavior: First, the agent verifies if it is located on a pixel: (1) not visited yet by another agent; (2) corresponding to the vessel pattern. To verify if the pixel belongs to a vessel pattern, the agent calculates the slope of the line constituted by its positions and the neighbors with positive gradient (Fig. 2 a)). Then, it analyses the gradient values profile on the perpendicular line to it (Fig. 2 b)), and if it corresponds to negativepositive-null-positive-negative values, the pixel belongs to a vessel edge. If it verifies the two conditions it determines the possible directions to follow, launches a NA on its position and moves to another white point, also randomly chosen. Moreover, it launches another SA on the parallel line to the one where it is initially located (Fig. 2 c)). For the determination of the directions to follow the agents look for the white points in their 8- 
neighbooring having a blue point in the 4-neighbooring. This blue point also has to belong to the 8-neighboors of the target pixel (Fig. 2 d)). The initially launched SA stops its behavior and disappears when all the white points were analyzed.

Node behavior: The node behavior is executed just one time. The NA launches FA in the directions given by the launcher agent (SA or FA) in the list of the possible directions to follow. If this list is empty it launches an $E A$, and then it establishes segments with those agents and keeps these segments in its segment list. After that, the NA stops its behavior.

Following behavior: At the beginning of the following behavior the agent checks its mail box to verify if there is another FA moving on the same line vessel but in opposite direction. In this case, they ask for a fusion process with each other by linking the respective NA. To construct the edge, the FA moves pixel per pixel and it stores the pixel position on it is located. The objective is to make sure that each position characterizing the edge portion which separates it from its neighbor can be approximated (using a threshold) by the segment connecting the two agents. The FAs move by determining the possible directions to follow as the SA do. If the FA has just one direction to follow, it checks if the segment formed between the position of its neighbor and its position is a valid segment, and if it is not, it launches another NA on its position and disappears. If it has more than one direction to follow it also launches a NA and disappears. After each movement, the FA sends a message to all the FAs in order to attempt a fusion.

End behavior: This behavior of the EA is to check if it is located on a blood vessel edge by analyzing the green intensity profile on the perpendicular direction to its segment. It just verifies if the profile is similar to a Gaussian shape as the blood vessels should be. If the profile does not fit a Gaussian curve, the EA disappear with its segment. If the profile fits a Gaussian curve the EA stops its behavior. This process is important to clean the small segments not belonging to the blood vessels, but to some noise and other imperfections of the background that still remains after the preprocessing phase. 


\section{Results and Discussion}

The proposed MAS model was implemented with MadKit [15] that is a generic multi-agent platform written in Java and built upon the AGR (Agent/Group/Role) organizational model. That is, the MadKit agents play roles in groups and thus create artificial societies.

The DRIVE dataset, a publicly available dataset developed by Niemeijer et al. [2], was used to test the proposed approach. It is compound of 40 images, in which 7 present signs of mild early diabetic retinopathy.

The performance of the edge reconstruction by the proposed MAS model depends directly on the image processing algorithms used in the preprocessing phase. It also depends of how the system interprets information resulting from this first phase of the approach. To measure the performance of the overall approach, it is important to compare the resulting image with the information detected by the Kirsch filter. Moreover, the differences between the resulting edge map image and the ground truth vessel map also present in the DRIVE dataset should be evaluated.

Results of the proposed approach applied to normal retinal images are shown in Fig. 3. These illustrate the original and resulting images where the proposed approach had the best and the worst performance respectively, with true positive rates of $88.9 \%$ and $71.7 \%$. The overall true positive rate for the DRIVE dataset was $83.1 \%$.

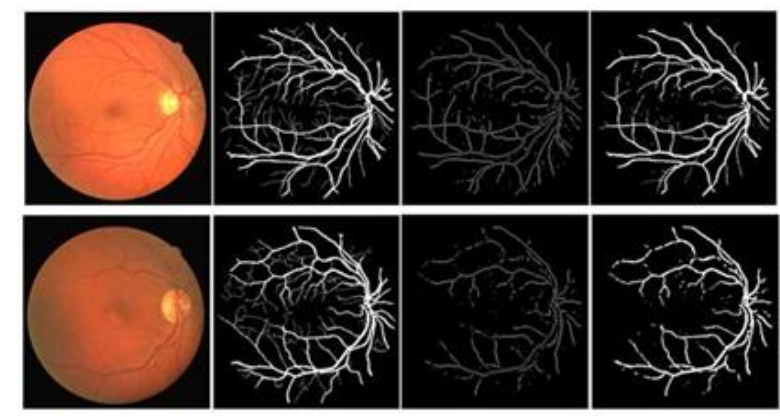

Fig. 3. Resulting images where the proposed approach had the best (above) and worst (below) performance in the DRIVE database. From left to right: original color fundus image; hand labeled image; edge detection by the MAS approach; the same image as the left one, but with a morphological posprocessing to better visualize the performance of the approach. 
By analyzing Fig. 1 a) and Fig. 3 (right one, above) it can be observed that the MAS reconstructed the edges of the most part of the vessels, especially of the thickest ones. Furthermore, they eliminated a large part of the pixels detected by the Kirsch filter that do not belong to the vessels. However, the MAS also cleaned some pixels belonging to the thinnest vessels, and some of these vessels were not detected at all, affecting the sensitivity values (Fig. 3). So, improvements have to be made in MAS model to deal with the small vessels.

Nevertheless, the experiments show that the use of a MAS model at the micro level could be an effective way to segment structures in complex images as the retinal images. In fact, through perception of the environment and local interactions, a simple agent organization can have as global behavior the detection of the most part of the retinal vasculature. The use of an improved version of the society of agents with some knowledge a priori about the retina proprieties, complemented with the use of some other traditional image processing algorithms could have the potential to develop a system to detect and differentiate all the anatomic and pathological structures of the fundus images. Actually, this approach is just a preliminary study of a large project where the goal is to develop a computer aided diagnosis system to be applied in regular screening programs to detect DR.

\section{Conclusion}

In this paper, a MAS approach is proposed where agents enrich a traditional edge detector algorithm allowing local processing adaptation and cooperative behaviors. The system is able to represent edges present on fundus image through segments, mainly the edges belonging to the vasculature.

It seems that a very efficient system for the diabetic retinopathy diagnosis could be built using a micro level approach, applying MAS mechanisms such as stigmergy for instance. Such an approach will overcome the classic image processing algorithms that are limited to macro results 
which cannot take into account the local characteristics of a complex image as the digital color fundus images are.

\section{References}

1. Hoover, A., Kouznetsova, V., Goldbaum, M.: Locating blood vessels in retinal images by piecewise threshold probing of a matched filter response. IEEE Trans. Med. Imag. 19(3), 203210 (2000).

2. Staal, J.J., Abrámoff, M.D., Niemeijer, M., Viergever, M.A., van Ginneken, B.: Ridge-based vessel segmentation in color images of the retina. IEEE Trans. Med. Imag. 23(4), 501-509 (2004).

3. Soares, J.V.B., Leandro, J.J., Cesar-Jr, R.M., Jelinek, H.F., Cree, M.J.: Retinal vessel segmentation using the 2-D Gabor wavelet and supervised classification. IEEE Trans. Med. Imag. 25(9), 1214-1222 (2006).

4. Ricci, E., Perfetti, R.: Retinal blood vessel segmentation using line operators and support vector classification. IEEE Trans. Med. Imag. 26(10), 1357-1365 (2007).

5. Mendonça, A., Campilho, A.: Segmentation of retinal blood vessel by combining the detection of centerlines and morphological reconstruction. IEEE Trans. Med. Imag. 25(9), 12001213 (2006).

6. Al-Diri, B., Hunter, A., Steel, D.: An active contour model for segmenting and measuring retinal vessels. IEEE Trans. Med. Imag. 28(9), (2009).

7. Mahdjoub, J., Guessoum, Z., Michel, F., Herbin, M.: A multi-agent approach for the edge detection in image processings. In: 4th European Workshop on Multi-Agent Systems, Lisbon, Portugal (2006).

8. Haroun, R., Boumghar, F., Hassas, S., Hamami, L.: A massive multi-agent system for brain MRI segmentation. In: Ishida, T., Gasser, L., Nakashima, H.: Massively Multi-Agent Systems I. LNCS, vol. 3446, pp. 174-186. Springer (2005).

9. Richard, N., Dojat, M., Garbay, C.: Automated segmentation of human brain MR images using a multi-agent approach. Artificial Intelligence in Medicine 30, 153-176 (2004).

10. Duchesnay, E., Montois, J.J., Jacquelet, Y.: Cooperative agents society organized as an irregular pyramid: A mammography segmentation application. Pattern recognition letters 24, 2435-2445 (2003).

11. Bovenkamp, E.G.P., Dijkstra, J., Bosch, J.G., Reiber, J.H.C.: Multi-agent segmentation of IVUS images. Pattern Recognition 37, 647-663 (2004).

12. Benamrane, N., Nassane, S.: Medical image segmentation by a multi-agent system approach. In: Petta, P., Müller, J.P., Klusch, M.,Georgeff, M.: Multiagent System Technologies. LNCS, vol.4687, pp. 49-60. Springer (2007).

13. Niemeijer, M., van Ginneken, B., Staal, J., Suttorp-Schulten, M., Abràmoff, M.D.: Automatic detection of red lesions in digital color fundus photographs. IEEE Trans. Med. Imag. 24(5), 584-592 (2005).

14. Kuwahara, M., Hachimura, K., Eiho, S., Kinoshita, M.: Digital Processing of Biomedical Images. Plenum Press, pp. 187-203, New York, NY (1976).

15. Madkit Homepage, http://www.madkit.org/. 